Pacific Journal of Mathematics

ADELE AND THE SPECTRUM OF COMPACT 


\section{ADELES AND THE SPECTRUM OF COMPACT NILMANIFOLDS}

\section{JEFFREY FoX}

Let $G$ be a nilpotent Lie group and $\Gamma$ a discrete cocompact subgroup of $G$. A basic problem in harmonic analysis is to determine the structure of $L^{2}(G / \Gamma)$. We apply adelic techniques to determine the decomposition of $L^{2}(G / \Gamma)$. To do so, we first develop a "rational" Kirillov theory for the adele group $G_{A}$. Once this is done, the decomposition and multiplicity formulas follow from elementary considerations.

Let $G$ be a locally compact group and $\Gamma \subset G$ a closed subgroup such that $G / \Gamma$ is compact. For $x \in G$, we can define $\lambda(x)$, a unitary operator on $L^{2}(G / \Gamma)$ by $(\lambda(x) f)(y)=f\left(x^{-1} y\right)$. The representation $x \rightarrow \lambda(x)$ is called the quasi-regular representation of $G$. A fundamental problem in representation theory is to decompose $\lambda$ into irreducible representations. A theorem of Fell [F] says that $\lambda$ will be discretely decomposable and each irreducible will occur with finite multiplicity. Thus we can write:

$$
L^{2}(G / \Gamma)=\sum_{\pi \in \operatorname{Sp}(\Gamma)} H(\pi) \otimes V_{\pi}
$$

where $0<\operatorname{dim}\left(V_{\pi}\right)<\infty$ for each $\pi \in \operatorname{Sp}(\Gamma)$. The first task is to determine $\operatorname{Sp}(\Gamma)$ as a subset of $\hat{G}$ and then to determine $\operatorname{dim}\left(V_{\pi}\right)$, the multiplicity with which $\pi$ occurs in $L^{2}(G / \Gamma)$. When $G$ is a solvable Lie group, one may use the reduction procedures of Howe [H2], Auslander-Brezin [B], and Fox [Fo]. In principle, then, the multiplicities can be computed, but the answers seem unsatisfying, and much work needs to be done in this area. For nilpotent Lie groups, the problem was first addressed by Moore [M], and later solved by Howe [H1] and Richardson [R]. The answer found by Howe and Richardson generalized the classical Frobenius reciprocity theorem for compact groups and provided a useful computational answer to the problem. Some time later, Corwin and Greenleaf [C-G] gave a beautiful solution, expressed in terms of canonical data attached to $\Gamma$ and the representation $\pi$, when $\Gamma$ satisfied some mild side conditions. 
In spite of the above work, and perhaps because of the success of the above authors, an important question remained unanswered. The question was first formulated for unipotent groups by Moore [M], and its importance was later emphasized by Howe [H1]. To describe the problem we need to recall the notion of the commensurability class of a discrete group $\Gamma$. We say that $\Gamma_{1}$ is commensurable with $\Gamma_{2}$ if $\Gamma_{1} \cap \Gamma_{2}$ has finite index in both $\Gamma_{1}$ and $\Gamma_{2}$. We will write $\{\Gamma\}$ for the class determined by $\Gamma$. The approach taken by the above authors is to pick a $\Gamma$ out of the class $\{\Gamma\}$ and then determine the decomposition of $L^{2}(G / \Gamma)$. The approach we shall take is to formulate and solve the problem for the class $\{\Gamma\}$; from that solution the decomposition of a particular $L^{2}(G / \Gamma)$ will follow as a simple corollary.

For nilpotent Lie groups, Moore formulated the problem and proved the fundamental theorem necessary to complete the solution. The class $\{\Gamma\}$ uniquely determines and is determined by, the structure of an affine algebraic group defined over $\mathbb{Q}$ on $G$. Let $G_{\mathbb{Q}}$ be the $\mathbb{Q}$ rational points of $G$. If $\mathbb{Q}_{A}$ is the ring of adeles of $\mathbb{Q}$ and $G_{A}$ the $\mathbb{Q}_{A}$ points of $G$ then we can embed $G_{\mathbb{Q}}$ diagonally into $G_{\mathrm{A}}$. The resulting quotient space, $G_{\mathrm{A}} / G_{\mathbb{Q}}$, is compact. Thus, $L^{2}\left(G_{\mathrm{A}} / G_{\mathbb{Q}}\right)$ is discretely decomposable. Moore has determined $\operatorname{Sp}\left(G_{\mathbb{Q}}\right)$ and showed that the multiplicity of each irreducible representation in $\operatorname{Sp}\left(G_{\mathbb{Q}}\right)$ is one. Once this decomposition is available, the local information follows readily, in principle. The representations of $G_{\mathrm{A}}$ can be constructed as infinite tensor products, and it is as a consequence of this construction that the local information is obtainable. However, to use the adele machinery effectively, we need a description of the representations occurring in $L^{2}\left(G_{\mathrm{A}} / G_{\mathbb{Q}}\right)$ that does not involve the infinite tensor product construction.

If $G$ is a nilpotent Lie group, models for the representations of $G_{A}$ that occur in $L^{2}\left(G_{\mathrm{A}} / G_{\mathrm{Q}}\right)$ can be constructed by applying the adele functor systematically, thereby obtaining a "rational" Kirillov theory for these groups. Once this is done, the multiplicity formulas of Howe-Richardson and Corwin-Greenleaf follow from elementary considerations. Moore's multiplicity one theorem also follows from a straightforward computation once the "rational" Kirillov theory has been constructed.

In $\S 1$ we describe the rational Kirillov theory for $G_{\mathrm{A}}$ and give a simple proof of Moore's multiplicity one theorem. In $\S 2$, we use the multiplicity one result to obtain the Howe-Richardson multiplicity formula and outline how the Corwin-Greenleaf results fit into the adele 
picture. We then obtain a sharp upper bound on the rate of growth of multiplicities (in terms of Plancherel density) for the generic representations of $G_{\infty}$ occurring in $L^{2}\left(G_{\infty} / \Gamma\right)$. It should be noted that by using Pukanszky's parameterization of all orbits occurring in $\mathfrak{g}^{*}$, it would be possible to produce a polynomial bound for all representations occurring in $L^{2}\left(G_{\infty} / \Gamma\right)$. (Consult [C-G2] for a very accessible description and application of this parameterization.)

What is most striking about the adelic approach to the multiplicity problem is the simplicity of the constructions and the ease with which the various multiplicity formulas follow from the adelic information. In particular, Moore's infinity tensor product construction is not needed.

The author would like to thank Richard Penney and Roger Zierau for conversations from which he profited greatly. The author would also like to thank the referee for many detailed comments that improved both the clarity and accuracy of this paper.

1. Rational Kirillov theory. Our basic references will be Weil's two books, [W-1] and [W-2]; from [W-2], we only need the first two chapters, which discuss the ideas of adelic geometry. Also, the reader might consult Tamagawa's brief survey of adeles ([T], p. 113). We now recall some definitions and notation. Given a prime $p$ of $\mathbb{Q}$, we can define a valuation ||$_{p}$ on $\mathbb{Q}$. If $x=p^{n} \frac{a}{b}$ with $b \in \mathbb{Z}$ and $a, b$ relatively prime to $p$, then $|x|_{p}=p^{-n}$. If we complete $\mathbb{Q}$ with respect to ||$_{p}$, we obtain $\mathbb{Q}_{p}$, a locally compact field. If || is the usual absolute value on $\mathbb{Q}$, then we will call || the valuation at the infinite prime and write ||$_{\infty}$. In this case, $\mathbb{Q}_{\infty}=\mathbb{R}$, the real numbers. For $p$, a finite prime $\mathbb{Q}_{p}$ will denote the closure of $\mathbb{Z}$ in $\mathbb{Q}_{p}$. Thus, $\mathbb{Z}_{p}$ is a compact open subgroup of $\mathbb{Q}_{p}$, which can also be described as $\mathbb{Z}_{p}=\left\{\left.x \in \mathbb{Q}_{p}|| x\right|_{p} \leq 1\right\}$. Given $x \in \mathbb{Q}$, we have the product formula:

$$
\prod_{p}|x|_{p}=1
$$

(Here, the product is over all primes, including the infinite one.)

We now recall the construction of the "ring of adeles" of $\mathbb{Q}$ (see [W-1], p. 59). Let $P$ be a finite set of primes that includes the infinite prime, and let $\mathbb{Q}(P)=\prod_{p \in P} \mathbb{Q}_{P} \times \prod_{p \notin P} \mathbb{Z}_{p}$ with the product topology. If $P_{1} \subseteq P_{2}$, then $\mathbb{Q}\left(P_{1}\right)$ is an open subring of $\mathbb{Q}\left(P_{2}\right)$. We set $\mathbb{Q}_{A}=$ $\bigcup_{P} \mathbb{Q}(P)$ with the inductive limit topology and call $\mathbb{Q}_{A}$ the adele ring of $\mathbb{Q}$. Given $x \in \mathbb{Q}_{A}$ we will write $x=\left(x_{\infty}, x_{p_{1}}, \ldots\right)$ if we need to exhibit $x$ in terms of coordinates. Given $q \in \mathbb{Q}$, we can write $q=p_{1}^{n_{1}} \cdots p_{e}^{n_{e}}$. 
Thus, if $p \neq p_{1}, \ldots, p_{e}$ we have $q \in \mathbb{Z}_{p}$. It follows that we can consider $\mathbb{Q}$ to be diagonally embedded in $\mathbb{Q}_{A}$. With this embedding, $\mathbb{Q}$ is a discrete cocompact subgroup of $\mathbb{Q}_{A}$. We can also view $\mathbb{Q}_{p}$ as being embedded in $\mathbb{Q}_{A}$ by $\mathbb{Q}_{p} \simeq\left\{x \in \mathbb{Q}_{A} \mid x=\left(0,0, \ldots, x_{p}, 0,0, \ldots\right)\right\}$. The statement that strong approximation holds for $\mathbb{Q}$ is simply that $\mathbb{Q}+\mathbb{Q} p$ is dense in $\mathbb{Q}_{A}$ for any $p\left([\mathbf{W}-\mathbf{1}], \mathbf{p}\right.$. 70). If we define $\mathbb{Q}_{f}=\{x \in$ $\left.\mathbb{Q}_{A} \mid x_{\infty}=0\right\}$, then $\mathbb{Q}_{A} \simeq \mathbb{Q}_{\infty} \times \mathbb{Q}_{f}$ as topological spaces. If $\psi$ is a character of $\mathbb{Q}_{A}$, then $\psi=\prod_{p} \psi_{p}$, with $\psi_{p}$ a character of $\mathbb{Q}_{p}$; and for all but a finite number of $p$ 's (to be abbreviated a.e. $p$ ), $\left.\psi_{p}\right|_{\mathbb{z}_{p}} \equiv 1$. A character $\psi$ of $\mathbb{Q}_{A}$ is called basic if $\psi$ is non-trivial but $\left.\psi\right|_{\mathbb{Q}} \equiv 1$. Note that strong approximation says that a basic character is determined by its restriction to $\mathbb{Q}_{p}$ (which is $\psi_{p}$ ) for any prime $p$. (See [W-1], part 1 , for more information on the above constructions.)

In [W-2], Chapter 1, Weil constructs the adele functor from the category of algebraic varieties defined over $\mathbb{Q}$ to the category of topological spaces. The construction is very similar to the construction of $\mathbb{Q}_{A}$ (we refer the reader to [W-2] for details and also to [T]). For $G$, a unipotent algebraic group defined over $\mathbb{Q}$, it will be useful to phrase the construction of $G_{A}$ in terms of restricted direct products. Moore ([M], pp. 163-64) gives a detailed account of this construction, a review of which follows.

Let $G_{\mathbb{Q}}$ be the $\mathbb{Q}$ rational points of $G$ and $\mathfrak{g}_{\mathbb{Q}}$ the Lie algebra of $G_{\mathbb{Q}}$. Let $X_{1}, \ldots, X_{n}$ be a basis of $\mathfrak{g}_{\mathbb{Q}}$ over $\mathbb{Q}$, and let $L$ be the $\mathbb{Z}$-span of $X_{1}, \ldots, X_{n}$; so $L$ is a lattice in $\mathfrak{g}_{\mathrm{Q}}$. By the Campbell-Baker-Hausdorff formula, there exists a polynomial $P: \mathfrak{g}_{\mathbb{Q}} \times \mathfrak{g}_{\mathbb{Q}} \rightarrow \mathfrak{g}_{\mathbb{Q}}$ such that $\exp (X)$. $\exp (X)=\exp (P(X, Y))$. It follows that for $G_{Q_{p}}$ multiplication is given by the same polynomial, but extended by continuity to $\mathfrak{g}_{\mathbb{Q}_{p}} \times \mathfrak{g}_{\mathbb{Q}_{p}}$. Let $L_{p}$ be the closure of $L$ in $\mathfrak{g}_{\mathfrak{Q}_{p}}$. If the coefficients of $P$ are in $\mathbb{Z}_{p}$ (which will happen for all but a finite number of primes), then $P: L_{p} \times L_{p} \rightarrow$ $L_{p}$ and $\exp \left(L_{p}\right)=K_{p}$ is a compact open subgroup of $G_{\mathbb{Q}_{p}}$. If we change the basis, then only a finite number of the $K_{p}$ 's will change; thus, we say that the $K_{p}$ 's are defined for a.e. $p$. Now we can form the restricted direct product of the $G_{\mathbb{Q}_{p}}$ with respect to the $K_{p}$ 's. Let $S$ be any finite set of primes containing those primes for which $K_{p}$ is not defined. Let $G(S)=\prod_{p \in S} G_{\mathrm{Q}_{p}} \times \prod_{p \notin S} K_{p}$ with the product topology. Then $G_{A}=\bigcup_{S} G(S)$ with the inductive limit topology.

Let $V$ be an algebraic variety defined over $\mathbb{Q}$; then $V_{A}$ will be the corresponding adele space attached to $V$. Given two varieties, $V_{1}$ and $V_{2}$, with a morphism $F: V_{1} \rightarrow V_{2}$ defined over $\mathbb{Q}$, there exists a canonical map $F_{A}:\left(V_{1}\right)_{A} \rightarrow\left(V_{2}\right)_{A}$ such that the following diagram 
commutes:

$$
\begin{array}{ccc}
\left(V_{1}\right)_{\mathbb{Q}} & \hookrightarrow & \left(V_{1}\right)_{A} \\
F \downarrow & & \downarrow F_{A} . \\
\left(V_{2}\right)_{\mathbb{Q}} & \hookrightarrow & \left(V_{2}\right)_{A}
\end{array}
$$

As for $\mathbb{Q}_{A}$, one can define $V_{p}, V_{f}, F_{p}$, and $F_{f}$ so that $V_{A}=V_{\infty} \times V_{f}$ and $F_{A}=F_{\infty} \times F_{j}$.

We now want to develop what might be called a rational Kirillov theory for unipotent algebraic groups defined over $\mathbb{Q}$. In what follows, we will use no subscript to denote the $\mathbb{Q}$ rational object and will employ the subscript $p$ or $A$ to denote the local or adele object. We fix the basic character $\psi$ of $\mathbb{Q}_{A}$ such that $\psi_{\infty}(x)=\exp (2 \pi i x)$.

Let $G$ be a unipotent algebraic group defined over $\mathbb{Q}$ with Lie algebra $\mathfrak{g}$. Let $\mathfrak{g}^{*}$ be the dual of $\mathfrak{g}$ and choose $l \in \mathfrak{g}^{*}$. A subalgebra $\mathfrak{h} \subseteq \mathfrak{g}$ is said to be a polarization for $l$ if $l([\mathfrak{h h}])=0$ and if $\mathfrak{h}$ is a maximal subspace with respect to this property. We let $H=\exp (\mathfrak{h})$ be the subgroup of $G$ with Lie algebra $\mathfrak{h}$ and $H_{A}$ represent the corresponding subgroup of $G_{A}$. We can define a character of $H_{A}$ (trivial on $H \subseteq H_{A}$ ) by the usual formula; for $h \in H_{A}$ :

$$
\chi_{l}(h)=\psi\left(l\left(\log _{A}(h)\right)\right) .
$$

DEFINITION 1.1. Let $\pi(l, H)=\operatorname{ind}_{H_{A}}^{G_{A}}\left(\chi_{l}\right)$.

LemMA 1.1. Let $l \in \mathfrak{g}^{*}$; then there exists a polarization $\mathfrak{h}$ for $l$ such that $\pi(l, H)$ is irreducible. Furthermore, up to equivalence, $\pi(l, H)$ is independent of $H$ and depends only on the $\operatorname{Ad}^{*}(G)$ orbit of $l$ in $\mathfrak{g}^{*}$. If $\mathscr{O}=\operatorname{Ad}^{*}(G) \cdot l \subseteq \mathfrak{g}^{*}$, then we will write $\pi_{\mathscr{O}}$ for a representative of the class determined by $\pi(l, H)$.

Proof. The proof is identical to the real case, so we will omit it. (However, one might consult [K1], p. 71, and [Wa], p. 326.)

As in the real case, the representations $\pi_{\mathscr{O}}, \mathscr{O} \in \mathfrak{g}^{*} / \operatorname{Ad}^{*}(G)$, will all be CCR and they will possess an appropriate orbital integral formula for the character. Before describing this, we need to recall the notion of a standard function (cf. [W-1], Ch. VII, §2). Let $C_{c}^{\infty}\left(G_{p}\right)$ be the $C^{\infty}$-function with compact support if $p$ is the infinite prime and the locally constant functions of compact support if $p$ is a finite prime. Let $\theta_{p}$ be the characteristic function of $K_{p}$ for those $p$ 's for which $K_{p}$ is defined (so $\theta_{p}$ is defined for a.e. $p$ ).

Definition 1.2. A function $\phi$ on $G_{A}$ is called standard if

$$
\phi\left(\left(x_{\infty}, x_{p_{1}}, \ldots\right)\right)=\prod_{p} \phi_{p}\left(x_{p}\right)
$$


with $\phi_{p} \in C_{c}^{\infty}\left(G_{p}\right)$ and $\phi_{p}=\theta_{p}$ for a.e. $p$. We denote by $C_{c}^{\infty}\left(G_{A}\right)$ the *-algebra (under convolution) of standard functions.

LEMMA 1.2. Let $\phi \in C_{c}^{\infty}\left(G_{A}\right)$; then $\phi$ is a finite sum of elements of the form $\phi_{1} * \phi_{2}$ with $\phi_{i} \in C_{\mathcal{C}}^{\infty}\left(G_{A}\right)$.

Proof. Write $\phi=\phi_{\infty} \times \phi_{f}$ with $\phi_{f}=\prod_{p \neq \infty} \phi_{p}$. By the DixmierMalliavin factorization theorem [D-M] $\phi_{\infty}=\sum_{i=1}^{k} \alpha_{i} * \beta_{i}$ with $\alpha_{i}$, $\beta_{i} \in C_{c}^{\infty}\left(G_{\infty}\right)$. Now consider $\phi_{f}=\prod_{p \in \infty} \phi_{p}$. For each $p, \phi_{p}$ is locally constant and thus invariant under an open compact subgroup of $G_{p}$, say $S_{p}$. Now for a.e. $p, \phi_{p}=\theta_{p}$, so we can take $S_{p}=K_{p}$ for a.e. $p$. If we set $S_{f}=\prod_{p} S_{p}$, then $\phi_{f}$ is invariant under $S_{f}$ and the characteristic function of $S_{f}$ is in $C_{c}^{\infty}\left(G_{f}\right)$. Now we have;

$$
\begin{aligned}
\phi_{f} * & \left(1 / \operatorname{vol}\left(S_{f}\right) \chi_{S_{f}}\right)(g) \\
= & \int_{G_{f}} \phi_{f}(g x)\left(1 / \operatorname{vol}\left(S_{f}\right) \chi_{S_{p}}(x)\right) d x=\phi_{f}(g) .
\end{aligned}
$$

If we set $\gamma=1 / \operatorname{vol}\left(S_{f}\right) \cdot \chi_{S_{f}}$, then $\gamma \in C_{c}^{\infty}\left(G_{f}\right)$ and we have:

$$
\begin{aligned}
\phi & =\phi_{\infty} \times \phi_{f}=\left(\sum \alpha_{i} * \beta_{i}\right) \times\left(\phi_{f} * \gamma\right) \\
& =\sum\left(\alpha_{i} \times \phi_{f}\right) *\left(\beta_{i} \times \gamma\right) .
\end{aligned}
$$

Suppose $\pi$ is an irreducible representation of $G_{A}$; then it is a consequence of Lemma 1.2 that $\pi(\phi)$ will be a trace class operator for all $\phi \in C_{c}^{\infty}\left(G_{A}\right)$ if $\pi(\phi)$ is a Hilbert Schmidt operator for all $\phi \in$ $C_{c}^{\infty}\left(G_{A}\right)$. To show $\pi(\phi)$ is a Hilbert Schmidt operator, it suffices to show that $\pi\left(\phi * \phi^{*}\right)$ is trace class. The point here is that we are reduced to deciding if a positive operator is trace class, which simplifies the computations. If $\pi=\operatorname{ind}_{H_{A}}^{G_{A}}\left(\chi_{l}\right)$, then we can compute $\operatorname{tr}\left(\pi\left(\phi * \phi^{*}\right)\right)$ using the standard computation for induced representations. Pukanszky's algorithm goes over almost word for word in the adele setting. After introducing some notation, we will describe how Pukanszky's algorithm can be adapted to this situation.

DEFINITION 1.3. Let $V$ be a non-singular algebraic variety defined over $\mathbb{Q}$. An algebraic differential form $\omega$ on $V$ is called a gauge form if $\operatorname{deg}(\omega)=\operatorname{dim}(V)$ and $\omega$ is everywhere holomorphic and non-zero (for a definition of holomorphic in the algebraic setting, see [L], pg. 189).

If $G$ is an affine algebraic group defined over $\mathbb{Q}$, then we have the usual definitions of right and left invariant differential forms on $G$. In 
particular, we say $G$ is unimodular if every left invariant gauge form is also right invariant. If $E \subseteq G$ is a subgroup of $G$ with $E$ and $G$ both unimodular, then there exists a gauge form on $G / E$ which is $G$ invariant ([W-2], p. 24). A standard induction on dimension of $n$ shows that unipotent groups are unimodular.

Given a gauge form $\omega$ on a non-singular algebraic variety $V$, Weil ([W-2], p. 21) shows how to construct measures $\omega_{p}$ on the $\mathbb{Q}_{p}$ points of $V$ for every $p$ and how to match up these measures to form measure on $V_{A}$. The measure on $V_{A}$ is essentially a product measure, which we denote by $(\omega)_{A}$. If $\omega$ is a left invariant gauge form on $G$, then $(\omega)_{A}$ is a left invariant measure on $G_{A}$. It follows that $G_{A}$ is unimodular if $G$ is unimodular. For unipotent groups, the basic case to consider is $G=\mathbb{Q}$. For each finite prime $p$, there is a unique Haar measure $(d X)_{p}$ on $\mathbb{Q}_{p}$ such that the volume of $\mathbb{Z}_{p}$ is one. We take on $\mathbb{R}=\mathbb{Q}_{\infty}$, the usual Lebesgue measure. For the gauge form $\omega=d X$ on $\mathbb{Q}$, the corresponding measure on $\mathbb{Q}_{A}$ is $(\omega)_{A}=\prod_{p}(d X)_{p}$.

If $G$ is a unipotent algebraic group and $E \subseteq G$ a Zariski closed subgroup with Lie algebra $\mathfrak{g}$, then we can construct a global crosssection for $G / E$ by means of a coexponential basis. Thus, we can find a basis $X_{1}, \ldots, X_{n}$ of $\mathfrak{g}$ such that $X_{1}, \ldots, X_{s}$ is a coexponential basis for $\mathfrak{e}$ in $g$ and $X_{s+1}, \ldots, X_{n}$ span $\mathfrak{e}$ over $\mathbb{Q}$. Consequently, we have the isomorphism $F: \mathbb{Q}^{s} \times \mathfrak{e} \rightarrow \mathfrak{g}$ defined by:

$$
F\left(\left(t_{1}, \ldots, t_{s}\right), X\right)=\exp \left(l_{1} X_{1}\right) \cdots \exp \left(t_{s} X_{s}\right) \cdot \exp (X) .
$$

Let $G$ be a unipotent algebraic group with Lie algebra $\mathfrak{g}$. The exponential map exp: $\mathfrak{g} \rightarrow G$ is an isomorphism of varieties. As in the real case (see, for instance, [Wa], p. 315), the pullback of a $G$ invariant gauge form on $G$ to $\mathfrak{g}$ via exp is a translation invariant gauge form on $\mathfrak{g}$. We fix a gauge form on $\mathfrak{g}$, say $d X$, and let $(d X)_{A}$ be the corresponding measure on $\mathfrak{g}_{A}$. If we put counting measure on the discrete cocompact subgroup $\mathfrak{g} \subseteq \mathfrak{g}_{A}$, then $(d X)_{A}$ is characterized by the fact that the volume of $\mathfrak{g}_{A} / \mathfrak{g}$ is one. The measure $(d X)_{A}$ is called the Tamagawa measure on $\mathfrak{g}_{A}$.

Definition 1.4. If $\phi \in C_{c}^{\infty}\left(G_{A}\right)$, we define the Fourier transform of $\phi$ as a function on $\mathfrak{g}_{A}^{*}$ by:

$$
\hat{\phi}(l)=\int_{\mathfrak{g}_{A}} \phi(X) \psi(l(X))(d X)_{A} .
$$

(Recall that $\psi$ is our fixed basic character of $\mathbb{Q}_{A}$.) 
Now $\mathfrak{g}_{A}^{*}$ also has a Tamagawa measure $(d l)_{A}$. It is easy to see that, with respect to this measure, Fourier inversion holds ([W-1], p. 113):

$$
\phi(0)=\int_{\left(\mathfrak{g}_{A}\right)^{*}} \hat{\phi}(l)(d l)_{A} .
$$

Let $\pi=\operatorname{ind}_{H_{A}}^{G_{A}}\left(\chi_{l}\right)$. We now recall Pukanszky's algorithm for computing $\operatorname{tr}(\pi(\phi))$ as an orbital integral. Let $\mathfrak{h}$ be the Lie algebra of $H$ and $X_{1}, \ldots, X_{n}$ basis of $\mathfrak{g}$ such that $X_{1}, \ldots, X_{s}$ is coexponential for $\mathfrak{h}$ in $\mathfrak{g}$ and $X_{s+1}, \ldots, X_{n}$ spans $\mathfrak{h}$. Set

$$
\Gamma=\left\{\gamma=\exp \left(t_{1} X_{1}\right) \cdots \exp \left(t_{s} X_{s}\right) \mid\left(t_{1}, \ldots, t_{s}\right) \in \mathbb{Q}^{s}\right\}
$$

and let $\mathfrak{h}^{\perp}=\left\{\lambda \in \mathfrak{g}^{*} \mid \lambda(X)=0 \forall X \in \mathfrak{h}\right\}$. If $\mathscr{O}=\operatorname{Ad}^{*}(G)(l) \subseteq \mathfrak{g}^{*}$, then $l+\mathfrak{h}^{\perp} \subseteq \mathscr{O}$. As in the real case, the map $L: \Gamma \times \mathfrak{h}^{\perp} \rightarrow \mathscr{O}$ defined by

$$
L(\gamma, \lambda)=\operatorname{Ad}^{*}(\gamma)(l+\lambda)
$$

is an isomorphism of varieties. If we identify $\Gamma$ with $\mathbb{Q}^{s}$ by

$$
\left(t_{1}, \ldots, t_{s}\right) \rightarrow \exp \left(t_{1} X_{1}\right) \cdots \exp \left(t_{s} X_{s}\right),
$$

then $\gamma$ has the gauge from $d \gamma=d t_{1} \wedge \cdots \wedge d t_{s}$. Let $X_{1}^{*}, \ldots, X_{s}^{*}$ be dual to $X_{1}, \ldots, X_{s}$; then if $\mathfrak{h}^{\perp}, \lambda=\sum_{i=1}^{s} u_{i} x_{i}^{*}$ with $u_{i} \in \mathbb{Q}$. This determines a gauge form $d \lambda=d u_{1} \wedge \cdots \wedge d u_{s}$ on $l+\mathfrak{h}^{\perp}$.

LEMMA 1.3. Let $l \in \mathfrak{g}^{*}, \mathfrak{h}$ a polarization for $l$ and $\pi=\operatorname{ind}_{H_{A}}^{G_{A}}\left(\chi_{l}\right)$. If $\phi \in C_{c}^{\infty}\left(G_{A}\right)$ is of the form $\phi=\alpha * \alpha^{*}$, then

$$
\operatorname{tr}(\pi(\phi))=\int_{(\Gamma)_{A}} \int_{\left(\mathfrak{h}^{\perp}\right)_{A}} \hat{\phi}\left(\operatorname{Ad}^{*}(\gamma)(l+\lambda)\right)(d \lambda)_{A}(d \gamma)_{A} .
$$

Proof. See [Pu2], p. 267.

Definition 1.5. Let $\omega_{\Gamma, H}$ be the gauge form on $\mathscr{O}$ such that $\omega_{\Gamma, H}=$ $L^{*}(d \lambda \wedge d \gamma)$.

Equation (1.10) can be written

$$
\operatorname{tr}(\pi(\phi))=\int_{\mathscr{O}_{A}} \hat{\phi}\left(\omega_{\Gamma, H}\right)_{A},
$$

where both sides are finite or both sides are infinite. To show that the right-hand side of (1.11) is finite, we need the following lemma:

LEMMA 1.4. Let $l \in \mathfrak{g}^{*}$ and $\mathscr{O}_{A}=\operatorname{Ad}^{*}\left(G_{A}\right)(l)=\left(\operatorname{Ad}^{*}(G)(l)\right)_{A}$; then $\mathscr{O}_{A}$ is closed in $\mathfrak{g}_{A}^{*}$.

Proof. Let $x_{n}=g_{n} \cdot l$ be a sequence in $\mathscr{O}_{A}$ such that $x_{n}$ converges to $y \in \mathfrak{g}_{A}^{*}$. Let $\bar{g}_{n}$ be the image of $g_{n}$ in $G_{A} / G$. Since $G_{A} / G$ is compact 
([M-T], p. 462), we can assume that $\bar{g}_{n}$ converges to $\bar{g}$ in $G_{A} / G$. Since the quotient map is open, we can find a sequence $z_{n} \in G_{A}$ such that $z_{n}$ converges to $z$ and $g_{n}=z_{n} \cdot w_{n}$ with $w_{n} \in G$. Now $\operatorname{Ad}^{*}\left(g_{n}\right) \cdot l$ converges and $z_{n}$ is convergent, so $\operatorname{Ad}^{*}\left(z_{n}^{-1} g_{n}\right) \cdot l=\operatorname{Ad}^{*}\left(w_{n}\right) \cdot l$ is convergent in $\mathfrak{g}_{A}^{*}$. But $\operatorname{Ad}^{*}\left(w_{n}\right) l \in \mathfrak{g}_{\mathrm{Q}}^{*} \subseteq \mathfrak{g}_{A}^{*}$ and $\mathfrak{g}_{\mathrm{Q}}^{*}$ is discrete; thus, if $n>N$, we have that $\operatorname{Ad}^{*}\left(w_{n}\right) l$ is the constant sequence. Therefore, $\operatorname{Ad}^{*}\left(g_{n}\right)(l)$ converges to $\operatorname{Ad}^{*}(z) \cdot \operatorname{Ad}^{*}\left(w_{N}\right)(l) \in \mathscr{O}_{A}$.

Let $\mathscr{O}_{A}=\mathscr{O}_{\infty} \times \mathscr{O}_{f}$; then $\mathscr{O}_{\infty} \subseteq \mathfrak{g}_{\infty}^{*}$ and $\mathscr{O}_{f} \subseteq \mathfrak{g}_{f}^{*}$ is also closed in $\mathfrak{g}_{f}^{*}$. Since $\hat{\phi}=\hat{\phi}_{\infty} \times \hat{\phi}_{f}$, we have:

(1.12) $\int_{\mathscr{O}_{A}} \hat{\phi} \cdot\left(\omega_{\Gamma, H}\right)_{A}=\left(\int_{\mathscr{O}_{\infty}} \hat{\phi}_{\infty} \cdot\left(\omega_{\Gamma, H}\right)_{\infty}\right) \times\left(\int_{\mathscr{O}_{j}} \hat{\phi}_{f} \cdot\left(\omega_{\Gamma, H}\right)_{f}\right)$.

It follows from [Pu], p. 267, that $\int_{\mathscr{O}_{A}} \hat{\phi}_{\infty} \cdot\left(\omega_{\Gamma, H}\right)_{\infty}$ is finite. As for the second factor, $\hat{\phi}_{f}$ has compact support in $\mathfrak{g}_{f}^{*}$. Since $\mathscr{O}_{f}$ is closed, $\operatorname{supp}\left(\hat{\phi}_{f}\right) \cap \mathscr{O}_{f}$ is compact in $\mathscr{O}_{f}$; from this, it follows that $\int_{\mathscr{O}_{f}} \hat{\phi}_{f} \cdot\left(\omega_{\Gamma, H}\right)_{f}$ is finite. We can summarize this with:

CoRollary 1.1. If $\pi=\operatorname{ind}_{H_{A}}^{G_{A}}\left(\chi_{l}\right)$ and $\phi \in C_{c}^{\infty}\left(G_{A}\right)$, then $\pi(\phi)$ is a trace class operator.

A priori, the gauge form $\omega_{\Gamma, H}$ depends on choices of a polarization $\mathfrak{h}$ for $l$ and a coexponential basis for $\mathfrak{h}$. However, given two $G$-invariant gauge forms, $\omega_{1}$ and $\omega_{2}$, on $\mathscr{O}$, there exists a constant $c \in \mathbb{Q}$ such that $\omega_{1}=c \omega_{2}$. It is now easy to check that $\left(\omega_{1}\right)_{A}=|c|_{A} \cdot\left(\omega_{2}\right)_{A}$ where $|c|_{A}=$ idéle norm of $c\left([\mathbf{W}-2]\right.$, p. 22). Since $c \in \mathbb{Q},|c|_{A}=1$ and $\left(\omega_{1}\right)_{A}=\left(\omega_{2}\right)_{A}$. We can now summarize our constructions with the following theorem.

THEOREM 1.1. Let $G$ be a unipotent algebraic group defined over $\mathbb{Q}$ with Lie algebra $\mathfrak{g}$. Given an $\operatorname{Ad}^{*}(G)$ orbit $\mathscr{O} \subseteq \mathfrak{g}^{*}$, we can associate with $\mathscr{O}$ an irreducible unitary representation $\left(\pi=\pi_{\mathscr{O}}\right)$ of $G_{A}$. Given $\phi \in C_{c}^{\infty}\left(G_{A}\right), \pi(\phi)$ is trace class and

$$
\operatorname{tr}(\pi(\phi))=\int_{\mathscr{O}_{A}} \hat{\phi} \cdot(\omega)_{A}
$$

where the canonical measure $(\omega)_{A}$ is obtained from any rational $G$ invariant gauge form on $\mathcal{O}$. 
THEOREM 1.2 (Moore). Let $G$ be a unipotent algebraic group defined over $\mathbb{Q}$. Let $\rho=\operatorname{ind}_{G}^{G_{A}}(1)$; then we have:

$$
\rho=\bigoplus_{\mathscr{O} \in \mathfrak{g}^{*} / \operatorname{Ad}^{*}(G)} \pi_{\mathscr{O}}
$$

Proof. We use the Selberg trace formula in conjunction with Poisson summation formula, as in [C-G]. Let $F$ be a fundamental domain of $G$ in $G_{A}$, so $\operatorname{vol}(F)=1$. Let $\phi \in C_{c}^{\infty}\left(G_{A}\right)$. Then we compute:

$$
\begin{aligned}
\operatorname{tr}(\rho(\phi)) & =\int_{F} \sum_{\gamma \in G} \phi\left(g^{-1} \gamma g\right) d \bar{g} \\
& =\int_{F} \sum_{X \in \mathfrak{g}} \phi\left(\operatorname{Ad}\left(g^{-1}\right)(X)\right) d \bar{g} \\
& =\int_{F} \sum_{l \in \mathfrak{g}^{*}} \hat{\phi}\left(\operatorname{Ad}^{*}(g)(l)\right) d \bar{g}
\end{aligned}
$$

In the last line, we use the Poisson summation formula for $\mathfrak{g} \subseteq \mathfrak{g}_{A}$ and $\mathfrak{g}^{*} \subseteq \mathfrak{g}_{A}^{*}$ (see [W-1], §2).

Let $S$ be a set of coset representatives for $\operatorname{Ad}^{*}(G)$ orbits in $\mathfrak{g}^{*}$. We then have:

$$
\begin{aligned}
\int_{F} \sum_{l \in \mathfrak{g}^{*}} \hat{\phi}\left(\operatorname{Ad}^{*}(g)(l)\right) d \bar{g} \\
=\sum_{l \in S}\left\{\int_{F} \sum_{\lambda \in G / G(l)} \hat{\phi}\left(\operatorname{Ad}^{*}(g \lambda)(l)\right) d \bar{g}\right\} \\
=\sum_{l \in S} \int_{G_{A} / G(l)} \hat{\phi}\left(\operatorname{Ad}^{*}(g)(l)\right) d \bar{g} .
\end{aligned}
$$

The measure on $G_{A} / G(l)$ is determined by giving the discrete group $G(l)$ counting measure. Finally, (1.16) becomes:

$$
\sum_{l \in S} \operatorname{vol}\left(G_{A}(l) / G(l)\right) \int_{G_{A} / G_{A}(l)} \hat{\phi}\left(\operatorname{Ad}^{*}(g)(l)\right) d \bar{g} .
$$

The measure on $G_{A} / G_{A}(l)$ is characterized by $\operatorname{vol}\left(G_{A}(l) / G(l)\right)$. This measure comes from a $G$-invariant gauge form on $G / G(l)$ if and only if $\operatorname{vol}\left(G_{A}(l) / G(l)\right)=1$. In this case, equations (1.13) and (1.17) yield:

$$
\operatorname{tr}(\rho(\phi))=\sum_{\mathscr{O} \in \mathfrak{g}^{*} / \mathrm{Ad}^{*}(G)} \operatorname{tr}\left(\pi_{\mathscr{O}}(\phi)\right) .
$$


2. Multiplicity formulas. In this section, we want to describe how the decomposition of $L^{2}\left(G_{A} / G\right)$ gives information about $L^{\infty}\left(G_{\infty} / \Gamma\right)$ when $G$ is a unipotent algebraic group defined over $\mathbb{Q}$. For more information on this process, one can consult [G-G-P], [Ma], and [S]. For unipotent groups, the proofs in [W-1] carry over.

Recall that if $E$ is a vector space over $\mathbb{Q}$, then $E_{\infty}+E$ is dense in $E_{A}([\mathbf{W}-1]$, p. 70$)$. If $G$ is an algebraic group defined over $\mathbb{Q}$, then strong approximation holds for $G$ if $G_{\infty} \cdot G$ is dense in $G_{A}$.

LEMMA 2.1. If $G$ is a unipotent group defined over $\mathbb{Q}$, then strong approximation holds for $G$.

Proof. Using that exp and log are isomorphisms, this follows from the corresponding fact for vector spaces.

Let $\Gamma$ be a discrete cocompact subgroup of $G_{\infty}$. For each finite prime $p$, let $\Gamma_{p}$ be the closure of $\Gamma$ in $G_{p}$. Then $\Gamma_{p}$ is a compact open subgroup of $G_{p}$, and for a.e. $p$. $\Gamma_{p}=K_{p}$ (see [M], pp. 163-64). We can associate with $\Gamma$ an open subgroup $K_{\Gamma}$ of $G_{A}$ by:

$$
K_{\Gamma}=G_{\infty} \times \prod_{p} \Gamma_{p}
$$

Conversely, suppose we are given a family of compact open subgroups $\Gamma_{p} \subset G_{p}$ such that $\Gamma_{p}=K_{p}$ for a.e. $p$ (equivalently, suppose we are given a compact open subgroup $\Gamma_{f}=\prod_{p \neq \infty} \Gamma_{p}$ of $G_{f}$ ); then we can form an open subgroup of $G_{A}, K=G_{\infty} \times \Gamma_{f}$. Let pr: $G_{A} \rightarrow G_{\infty}$ be the projection onto the first factor. If $\Gamma_{K}=\operatorname{pr}(K \cap G)$, then $\Gamma_{K}$ is a discrete cocompact subgroup of $G_{\infty}$. The two correspondences $\Gamma \rightarrow K_{\Gamma}$ and $K \rightarrow \Gamma_{K}$ are inverses of each other. The proofs of these facts are identical to Theorem 1 in [W-1], p. 84 .

Now fix a $\Gamma \subseteq G_{\infty}$; then we can define a natural map $T$ from $L^{2}\left(G_{\infty} / \Gamma\right)$ to $L^{2}\left(G_{A} / G\right)$. Strong approximation says $G_{A}=K_{\Gamma} \cdot G$; thus, if $x \in G_{A}$, we can write $x=\left(g_{\infty}, g_{f}\right) \cdot \gamma$ with $\left(g_{\infty}, g_{f}\right) \in K_{\Gamma}$ and $\gamma \in G$. If $\phi \in L^{2}\left(G_{\infty} / \Gamma\right)$, define:

$$
T(\phi)(x)=T(\phi)\left(\left(g_{\infty}, g_{f}\right) \cdot \gamma\right)=\phi\left(g_{\infty}\right) .
$$

If $x=\left(g_{\infty}, g_{f}\right) \cdot \gamma=\left(\bar{g}_{\infty}, \bar{g}_{f}\right) \cdot \bar{\gamma}$, then $\left(g_{\infty}^{-1} \bar{g}_{\infty}, g_{f}^{-1} \bar{g}_{f}\right)=\bar{\gamma} \gamma^{-1} \in G \cap K$; so $g_{\infty}^{-1} \bar{g}_{\infty} \in \Gamma$ and $\phi\left(g_{\infty}\right)=\phi\left(\bar{g}_{\infty}\right)$. Thus, $T$ is well-defined.

Next we want to show that $T(\phi)$ is in $L^{2}\left(G_{\mathrm{A}} G_{Q}\right)$. Since $G_{\mathrm{A}}=K_{\Gamma} \cdot G_{Q}$ we have:

$$
\int_{G_{\mathrm{A}} / G_{Q}}|T(\phi)|^{2} \leq \int_{K_{\Gamma} / K_{\Gamma} \cap G_{Q}}|T(\phi)|^{2} .
$$


Set $\bar{\Gamma}=K_{\Gamma} \cap G_{Q}=\{(\gamma, \gamma, \ldots) \mid \gamma \in \Gamma\}$ and let $h \in C_{c}\left(K_{\Gamma}\right)$. We have by our normalizations of measures:

$$
\int_{K_{\Gamma} / \bar{\Gamma}}\left(\sum_{\bar{\gamma} \in \bar{\Gamma}} h(g \bar{\gamma})\right)=\int_{K_{\Gamma}} h(g) .
$$

Next suppose $h$ is $\Gamma_{f}$ invariant. Then $\exists f \in C_{c}\left(G_{\infty}\right)$ such that $f\left(q_{\infty}\right)=$ $h(q)$ for all $q \in K_{\Gamma}$, thus we have:

$$
\int_{K_{\Gamma}} h(q)=\operatorname{vol}\left(\Gamma_{f}\right) \int_{G_{\infty}} f\left(q_{\infty}\right)=\operatorname{vol}\left(\Gamma_{f}\right) \int_{G_{\infty} / \Gamma}\left(\sum_{\gamma \in \Gamma} f\left(q_{\infty} \cdot \gamma\right),\right.
$$

$$
\int_{K_{\Gamma}} h(q)=\int_{K_{\Gamma} / \bar{\Gamma}}\left(\sum_{\bar{\gamma} \in \Gamma} h(q \bar{\gamma})\right)=\int_{K_{\Gamma} / \bar{\Gamma}} T\left(\sum_{\gamma \in \Gamma} f\left(q_{\infty} \cdot \gamma\right)\right) .
$$

Thus (2.5) and (2.6) yield:

$$
\operatorname{vol}\left(\Gamma_{f}\right) \int_{G_{\infty} / \Gamma} \phi=\int_{K_{\Gamma} / \bar{\Gamma}} T(\phi)
$$

Since $T\left(|\phi|^{2}\right)=|T(\phi)|^{2}$ we get:

$$
\int_{G_{\mathbf{A}} / G_{Q}}|T(\phi)|^{2} \leq \int_{K_{\Gamma} / \bar{\Gamma}}|T(\phi)|^{2}=\operatorname{vol}\left(\Gamma_{f}\right) \cdot \int_{G_{\infty} / \Gamma}|\phi|^{2} .
$$

Thus $T$ is a continuous mapping of $L^{2}\left(G_{\infty} / \Gamma\right)$ into $L^{2}\left(\Gamma_{f} / G_{A} \backslash G_{Q}\right)$. To see that $T$ is onto we produce an inverse. Let $\psi \in L^{2}\left(\Gamma_{f} / G_{A} \backslash G_{Q}\right)$; then define $(S \psi)\left(q_{\infty}\right)=\psi\left(\left(q_{\infty}, 1,1, \ldots\right)\right)$. We note that $S \psi$ is left $\Gamma$ invariant, for we have:

$$
\begin{aligned}
(S \psi)\left(q_{\infty} \cdot \gamma\right) & \left.=\psi\left(q_{\infty} \gamma, 1, \ldots\right)\right) \\
& =\psi\left(\left(1, \gamma^{-1}, \gamma^{-1}, \ldots\right)\left(q_{\infty}, 1,1, \ldots\right)(\gamma, \gamma, \gamma, \ldots)\right) \\
& =\psi\left(\left(q_{\infty}, 1,1, \ldots\right)\right)
\end{aligned}
$$

where we have used $\left(1, \gamma^{-1}, \gamma^{-1}, \ldots\right) \in \Gamma_{f}$ for all $\gamma \in \Gamma$.

Similar computations as for $T$, show that $S$ maps $L^{2}\left(\Gamma_{f} / G_{A} \backslash G_{Q}\right)$ continuously into $L^{2}\left(G_{\infty} / \Gamma\right)$.

Recall that $\rho$ is left translation by $G_{A}$ on $L^{2}\left(G_{A} / G\right)$ and $\lambda$ is left translation by $G_{\infty}$ on $L^{2}\left(G_{\infty} / \Gamma\right)$. If $g=\left(g_{\infty}, g_{f}\right) \in K_{\Gamma}$, then we have:

$$
\rho(g) T(\phi)=T\left(\lambda\left(g_{\infty}\right) \phi\right) .
$$


LEMMA 2.2. Let $\bar{\rho}$ denote the restriction of $\rho$ to the subgroup $K_{\Gamma}=$ $G_{\infty} \times \Gamma_{f} \subseteq G_{A}$ acting on the invariant subspace $L^{2}\left(\Gamma_{f} / G_{A} \backslash G\right)$. If $S_{p}(\Gamma)$ denotes the representations of $G_{\infty}$ that occur with positive multiplicity in $L^{2}\left(G_{\infty} / \Gamma\right)$, then we have:

$$
\bar{\rho}=\sum_{\sigma \in S_{p}(\Gamma)} m(\sigma)(\sigma \otimes 1) .
$$

(Here $\sigma \otimes 1$ is the representation of $K_{\Gamma}$ given by $(\sigma \otimes 1)\left(g_{\infty}, g_{f}\right)=$ $\sigma\left(g_{\infty}\right)$.)

Proof. This is just a restatement of 2.10 .

Let $\pi$ be an irreducible representation of $G_{A}$ occurring in $L^{2}\left(G_{A} / G\right)$. Since $G_{A}=G_{\infty} \times G_{f}$ and $G_{\infty}$ is a type 1 group, there exist irreducible representations $\pi_{\infty}, \pi_{f}$ of $G_{\infty}, G_{f}$ such that $\pi=\pi_{\infty} \times \pi_{f}$. Theorem 1.2 and formula (1.12), tell us that $\pi$ is the only irreducible representation of $G_{A}$ occurring in $L^{2}\left(G_{A} / G\right)$ with the $\infty$-factor being $\pi_{\infty}$. If $\left.\pi_{f}\right|_{\Gamma_{f}}=$ $\sum_{\tau \in \Gamma_{f}} \mu\left(\pi_{f}, \tau\right) \tau$, then $\left.\pi\right|_{K_{\Gamma}}=\sum_{\tau \in \widehat{\Gamma}_{f}} \mu\left(\pi_{f}, \tau\right)\left(\pi_{\infty} \otimes \tau\right)$. If we consider just the trivial representation of $\Gamma_{f}$, then we have that $\pi_{\infty} \otimes 1$ occurs in $L^{2}\left(\Gamma_{f} \backslash G_{A} / G\right)$ with multiplicity $\mu\left(\pi_{f}, 1\right)$. We can summarize the above discussion with the following lemma.

LemMA 2.3. Let $G$ be a unipotent algebraic group defined over $\mathbb{Q}$. Let $\Gamma$ be a discrete, cocompact subgroup of $G_{\infty}$ and $\Gamma_{f}=\prod_{p_{p} \neq \infty} \Gamma_{p}$ the compact subgroup of $G_{A}$ corresponding to $\Gamma$. Let $\pi=\pi_{\infty} \times \pi_{f}$ be an irreducible representation of $G_{A}$ that occurs in $L^{2}\left(G_{A} / G\right)$. If $m\left(\pi_{\infty}\right)$ denotes the multiplicity of $\pi_{\infty}$ in $L^{2}\left(G_{\infty} / \Gamma\right)$ and $\mu\left(\pi_{f}\right)$ denotes the multiplicity of the trivial representation of $\Gamma_{f}$ in $\left.\pi_{f}\right|_{\Gamma_{f}}$, then:

$$
m\left(\pi_{\infty}\right)=\mu\left(\pi_{f}\right)
$$

In particular, for an irreducible representation $\pi_{\infty}$ of $G_{\infty}$ to occur in $L^{2}\left(G_{\infty} / \Gamma\right)$, it is necessary that $\pi=\pi_{\infty} \times \pi_{f}$ for some $\pi$ of Theorem 1.2 .

Since we have such a nice model for $\pi_{\mathscr{O}}=\operatorname{ind}_{H_{A}}^{G_{A}}\left(\chi_{l}\right)$, we can easily use Lemma 2.3 to compute the decomposition of $L^{2}\left(G_{\infty} / \Gamma\right)$. We see that $\operatorname{ind}_{H_{A}}^{G_{A}}\left(\chi_{l}\right)=\operatorname{ind}_{H_{\infty}}^{G_{\infty}}\left(\chi_{l}\right) \times \operatorname{ind}_{H_{f}}^{G_{f}}\left(\chi_{l}\right)$, so the representation $\pi_{f}$ of Lemma 2.3 is an induced representation. Thus, we can apply the Mackey subgroup theorem to compute:

$$
\left.\pi_{f}\right|_{\Gamma_{f}}=\sum_{\pi \in H_{f} / G_{f} \backslash \Gamma_{f}} \operatorname{ind}_{x H_{f} x^{-1} \cap \Gamma_{f}}^{\Gamma_{f}}\left(\chi_{l}\right)^{x} .
$$


We need to remark on two points in equation (2.13). First, since the subgroup $\Gamma_{f}$ is open in $G_{f}$, we have that $\Gamma_{f}$ and $H_{f}$ are regularly related, $\Gamma_{f} \backslash G_{f} / H_{f}$ being discrete. Let $\bar{G}$ be the projection of $G \subseteq G_{\mathrm{A}}$ into $G_{f} \subseteq G_{\mathrm{A}}$, thus $\bar{G}=\left\{(1, x, x, \ldots) \mid x \in G_{Q}\right\}$. It follows from [W-1], pg. 83 , Corollary 1 , that $\bar{G}$ is dense in $G_{f}$, thus we have $G_{f}=\Gamma_{f} \cdot \bar{G}$. Therefore, (2.13) says:

$$
\mu\left(\pi_{f}\right)=\sum_{\substack{x \in H_{f} / G_{f} \backslash \Gamma_{f} \\ x \in \bar{G}}} \operatorname{dim} \operatorname{Hom}_{\Gamma_{f}}\left(1, \operatorname{ind}_{x H_{f} x^{-1} \cap \Gamma_{f}}^{\Gamma_{f}}\left(\chi_{l}\right)^{x}\right) .
$$

Now $\Gamma_{f}$ is a compact group, so we can use the classical Frobenius reciprocity theorem for compact groups to conclude:

$$
\operatorname{Hom}_{\Gamma_{f}}\left(1, \operatorname{ind}_{x H_{f} x^{-1} \cap \Gamma_{f}}^{\Gamma_{f}}\left(\chi_{l}\right)^{x}\right) \simeq \operatorname{Hom}_{x H_{f} x^{-1} \cap \Gamma_{f}}\left(1,\left(\chi_{l}\right)^{x}\right) .
$$

In what follows, we will assume that $x$ is the identity. Thus, we are in the situation where:

$$
\left.\psi \circ l\left(\log _{f}(\cdot)\right)\right|_{H_{f} \cap \Gamma_{f}} \equiv 1 \quad \text { and }\left.\quad \psi \circ l\left(\log _{A}(\cdot)\right)\right|_{H_{\mathbb{Q}}} \equiv 1 .
$$

If $\gamma \in H_{\mathbb{Q}}$, then we have $\psi_{\infty} \circ l\left(\log _{\infty}(\gamma)\right)=\psi_{f} \circ l\left(\log _{f}(\gamma)\right)^{-1}$; and if $\gamma \in H_{f} \cap \Gamma_{f}$, then $\psi_{f} \circ l\left(\log _{f}(\gamma)\right)^{-1}=1$ by 2.16 , so $\psi_{\infty} \circ l\left(\log _{\infty}(\gamma)\right)=1$. Thus, we see that:

$$
\left.\left(\chi_{l}\right)_{f}\right|_{H_{f} \cap \Gamma_{f}} \equiv 1 \quad \text { iff }\left.\left(\chi_{l}\right)_{\infty}\right|_{H_{\infty} \cap \Gamma} \equiv 1 .
$$

Consequently, we have:

(2.18) $\operatorname{dim} \operatorname{Hom}_{x H_{f} x^{-1} \cap \Gamma_{f}}\left(1,\left(\chi_{l}\right)^{x}\right)= \begin{cases}1 & \text { if }\left.\left(\chi_{l}\right)_{\infty}\right|_{x H x^{-1} \cap \Gamma}=1, \\ 0 & \text { otherwise. }\end{cases}$

We can summarize the discussion with the following theorem.

THEOREM 2.1 (Howe-Richardson). An irreducible representation $\pi$ of $G_{\infty}$ occurs in $L^{2}\left(G_{\infty} / \Gamma\right)$ if and only if there exist $l \in \mathfrak{g}_{Q}^{*}$ and a rational polarization $H$ for $\left(\chi_{l}\right)_{\infty}$ such that $\pi=\operatorname{ind}_{H_{\infty}}^{G_{\infty}}\left(\left(\chi_{l}\right)_{\infty}\right)$ and $\left.\left(\chi_{l}\right)_{\infty}\right|_{H_{\infty} \cap \Gamma} \equiv$ 1. The multiplicity of $\pi_{\infty}$ in $L^{2}\left(G_{\infty} / \Gamma_{\infty}\right)$ is given by the number of rational double cosets in $H_{\infty} \backslash G_{\infty} / \Gamma$ such that $\left.\left(\chi_{l}\right)_{\infty}^{x}\right|_{x H_{\infty} x^{-1} \cap \Gamma}$ $\equiv 1$.

We now indicate briefly how the results of Corwin-Greenleaf [C-G] fit into the present picture. We restrict ourselves to the case where $\Lambda=\log (\Lambda)$ is a lattice in $\mathfrak{g}$; such $\Gamma$ 's are called lattice subgroups. In this case, $\log \left(\Gamma_{p}\right)=\Lambda_{p} \subseteq \mathfrak{g}_{p}$ is a compact open $\mathbb{Z}_{p}$ module of $\mathfrak{g}_{p}$ 
([M], p. 161), so $\prod_{p} \Lambda_{p}=\Lambda_{f}$ is a compact open subgroup of $\mathfrak{g}_{f}$. Let $\phi \in C_{c}^{\infty}\left(G_{A}\right)$ be given by $\phi=\phi_{1} \times \phi_{2}$, with $\phi_{2}$ the characteristic function $\Gamma_{f}$ (which we identify with the characteristic function of $\Lambda_{f}$ via the exponential map). We have:

$$
\operatorname{tr}(\pi(\phi))=\operatorname{tr}\left(\pi_{\infty}\left(\phi_{1}\right)\right) \cdot \operatorname{tr}\left(\pi_{f}\left(\phi_{2}\right)\right) .
$$

If $P$ is the projection onto the space of $\Gamma_{f}$ fixed vectors in $H\left(\pi_{f}\right)$, it is well known that $P=\operatorname{vol}\left(\Gamma_{f}\right)^{-1} \cdot \pi_{f}\left(\phi_{2}\right)$. Thus,

$$
\operatorname{tr}\left(\pi_{f}\left(\phi_{2}\right)\right)=\operatorname{vol}\left(\Gamma_{f}\right) \cdot \operatorname{tr}(P)=\operatorname{vol}\left(\Gamma_{f}\right) \mu\left(\pi_{f}\right)=\operatorname{vol}\left(\Gamma_{f}\right) m\left(\pi_{\infty}\right) .
$$

To compute $m\left(\pi_{\infty}\right)$, we only need apply the character formula for $\operatorname{tr}(\pi(\phi))$. Since $\Gamma$ is a lattice subgroup, this is particularly easy, since $\hat{\phi}=\hat{\phi}_{1} \times \hat{\phi}_{2}$ and $\hat{\phi}_{2}=\operatorname{vol}\left(\Lambda_{f}\right) \cdot\left(\right.$ characteristic function of $\Lambda_{f}^{\perp}$ in $\left.\mathfrak{g}^{*}\right)$. (See [W-1], p. 107.) We then get:

$$
\operatorname{tr}(\pi(\phi))=\left(\int_{\mathscr{O}_{\infty}} \hat{\phi}_{\infty} d \mu_{\infty}\right) \cdot\left(\int_{\mathscr{O}_{f}} \hat{\phi}_{f} d \mu_{f}\right) .
$$

If we break $\mathscr{O}_{f} \cap \Lambda_{f}^{\perp}$ into a sum of $\Gamma_{f}$ orbits and evaluate the resulting integrals, we obtain the formula of Corwin and Greenleaf ([C-G], p. 12). We omit the details.

Next we will use the trace formula (1.12) to obtain a sharp upper bound for the rate of growth of the multiplicities of representations in the spectrum of $\Gamma$. When the representations are square-integrable mod the center, the estimate becomes exact and gives the Moore-Wolf multiplicity formula [M-W].

LeMmA 2.4. Let $X_{1}, \ldots, X_{n}$ be a Jordan-Hölder basis for $\mathfrak{g}$. Thus, if $\mathfrak{g}_{i}=\operatorname{span}_{\mathbb{Q}}\left\{X_{1}, \ldots, X_{i}\right\}$, then $\mathfrak{g}_{i}$ is an ideal of $\mathfrak{g}$. Let $l_{1}, \ldots, l_{n}$ be the corresponding dual basis of $\mathfrak{g}^{*}$. There exist complementary subsets $S, T$ of $\{1,2, \ldots, n\}$ and an $\operatorname{Ad}^{*}(G)$ invariant Zariski-open dense subset $\mathscr{W}$ of $W=\operatorname{span}_{\mathbb{Q}}\left\{l_{j} \mid j \in T\right\}$ such that if $V=\operatorname{span}\left\{l_{j} \mid j \in S\right\}$, then:

(1) for almost every $\operatorname{Ad}^{*}(G)$ orbit $\mathscr{O} \subseteq \mu^{*}, \mathscr{O} \cap \mathscr{W}$ has only one element (if $\mathscr{O} \cap \mathscr{W}=\{l\}$, then we will write $\mathscr{O}_{l}$ for $\left.\mathscr{O}\right)$;

(2) for all $l \in \mathscr{W}$, there exists a polynomial map $P_{l}: V \rightarrow W$ with $\mathscr{O}_{l}=\operatorname{graph}\left(P_{l}\right) ;$ and

(3) the map $l \rightarrow P_{l}$ is rational in $l$.

REMARK. Almost every orbit means a non-empty Zariski open subset of $\mathfrak{g}^{*}$.

Proof. See [Pu1], p. 55. 
The basis $X_{i}, i \in S$ for $V$ determines a gauge form on $V$, which we denote by $d X$. Let $R_{k}: \mathscr{O}_{l} \rightarrow V$ be defined as follows:

$$
R_{l}\left(\left(x, P_{l}(x)\right)\right)=x \text {. }
$$

Then we can consider the gauge form on $\mathscr{O}_{l}$ defined by $R_{l}^{*}(d x)=\omega_{l}$.

LEMMA 2.5. The gauge form $\omega_{l}=R_{l}^{*}(d x)$ is a $G$ invariant form on $\mathscr{O}_{l}$.

Proof. [Pu1], p. 54.

Corollary 2.1. The canonical measure on $\left(\mathscr{O}_{l}\right)_{A}$ is given by $\left(\omega_{l}\right)_{A}$.

Let $\pi_{l}$ be the irreducible representation of $G_{A}$ associated with $\mathscr{O}_{l}$. If $\phi \in C_{c}^{\infty}\left(G_{A}\right)$, then it follows from Corollary 2.1 that:

$$
\operatorname{tr}\left(\pi_{l}(\phi)\right)=\int_{(V)_{A}} \hat{\phi}\left(x+P_{l}(x)\right)(d x)_{A} .
$$

If $\phi=\phi_{\infty} \times \phi_{f}$, then (2.22) becomes:

$$
\begin{aligned}
\operatorname{tr}\left(\pi_{l}(\phi)\right)= & \int_{(V)_{\infty}} \hat{\phi}_{\infty}\left(x+P_{l}(x)\right)(d x)_{\infty} \\
& \times \int_{V_{f}} \hat{\phi}_{f}\left(x+P_{l}(x)\right)(d x)_{f} .
\end{aligned}
$$

It is well known that there exists a polynomial function $l \rightarrow P f(l)$ on $\mathscr{W}$ such that ([C], Theorem 2):

$$
\operatorname{tr}\left(\pi_{\infty}\left(\phi_{\infty}\right)\right)=|P f(l)|_{\infty}^{-1} \int_{(V)_{\infty}} \hat{\phi}_{\infty}\left(x+P_{l}(x)\right)(d x)_{\infty}
$$

It follows from (2.23) and (2.24) that:

$$
\operatorname{tr}\left(\pi_{f}\left(\phi_{f}\right)\right)=|P f(l)|_{\infty} \int_{(V)_{f}} \hat{\phi}_{f}\left(x+P_{l}(x)\right)(d x)_{f} .
$$

(Since $(P f)(l)$ is rational if $l$ is rational, we have $|P f(l)|_{A}=1$, where $|X|_{A}=\prod_{p}\left|X_{p}\right|_{p}$ is the idele norm, or $\left.|P f(l)|_{\infty}=|P f(l)|_{f}^{-1} \cdot\right)$

Now suppose $\Gamma \subseteq G_{\infty}$ is a lattice subgroup of $G_{\infty}$, and set $\Lambda=$ $\log (\Gamma)$. Let $\phi_{f}$ be the characteristic function of $\Lambda_{f}$. We saw before that $\operatorname{tr}\left(\left(\pi_{l}\right)_{f}\left(\phi_{f}\right)\right)=\operatorname{vol}\left(\Lambda_{f}\right) m\left(\pi_{\infty}\right)$. Since $\phi_{f}$ is the characteristic function of $\Lambda_{f}, \hat{\phi}_{f}=\operatorname{vol}\left(\Lambda_{f}\right) \cdot K_{\Lambda_{f}^{\perp}}$, where $K_{\Lambda_{f}^{\perp}}$ is the characteristic function of $\Lambda_{f}^{\perp}=\left\{\lambda \in \mathfrak{g}_{f}^{*} \mid \psi_{f}\left(\lambda\left(\Lambda_{f}\right)\right)=1\right\}$. Applying (2.25), we have:

(2.26) $m\left(\left(\pi_{l}\right)_{\infty}\right)=1 / \operatorname{vol}\left(\Lambda_{f}\right)$

$$
\cdot \operatorname{vol}\left(\Lambda_{f}\right)|P f(l)|_{\infty} \int_{(V)_{f}} K_{\Lambda_{f}^{\perp}}\left(x+P_{l}(x)\right)(d x)_{f}
$$


For $K_{\Lambda_{f}^{\perp}}\left(x+P_{l}(x)\right)$ to be one, we need at least that $x \in V_{f} \cap \Lambda_{f}^{\perp}$; so we get:

$$
\int_{V_{f}} K_{\Lambda_{f}^{\perp}}\left(x+P_{l}(x)\right)(d x)_{f} \leq \operatorname{vol}\left(\Lambda_{f}^{\perp} \cap V_{f}\right)
$$

We can summarize the above with:

LEMMA 2.2. Let $\Gamma \subseteq G_{\infty}$ be a lattice subgroup of $G_{\infty}$. Let $\mathscr{W}$ be as above. Then for every $l \in \mathscr{W}$ we have:

$$
m\left(\left(\pi_{l}\right)_{\infty}\right) \leq A|P f(l)|_{\infty}, \quad \text { where } A=\operatorname{vol}\left(\Lambda_{f}^{\perp} \cap V_{f}\right) .
$$

ReMARKs. (1) It is well known that $|P f(l)|_{\infty}$ is Plancherel density with respect to the appropriate coordinates (see [C], p. 6, or [K2]).

(2) Suppose $G$ has square-integrable representations. Then, if $\mathfrak{z}=$ center of $\mathfrak{g}$, we can take $V \simeq \mathfrak{z}^{\perp}, W \approx \mathfrak{z}^{*}$, and $P_{l}(l) \equiv l[\mathbf{M}-\mathbf{W}$ ]. Applying formula (2.26) yields:

$$
m\left(\left(\pi_{l}\right)_{\infty}\right)= \begin{cases}|P f(l)| & \text { if } l \in(\mathfrak{z} \cap \Lambda)^{\perp} \\ 0 & \text { otherwise. }\end{cases}
$$

\section{REFERENCES}

[Bo] A. Borel, Algebraic Groups, Benjamin, New York, 1969.

[B] J. Brezin, Harmonic Analysis on Compact Solvmanifolds, LNM \#602, Springer-Verlag, New York, 1977.

[C] L. Corwin, A representation theoretic criterion for local solvability of leftinvariant differential operators on nilpotent lie groups, Trans. Amer. Math. Soc., 264 (1981), 113-120.

[C-G1] L. Corwin and F. Greenleaf, Character formula and spectra of compact nilmanifolds, J. Funct. Anal., 221 (1976), 123-154.

[C-G2] - Direct integral decompositions and multiplicities for induced representations of nilpotent Lie groups, (preprint).

[D-M] J Dixmier and P. Malliavin, Factorization de fonctions et de vecteurs indefiniment differentiables, Rulf. Sc. Math., $2^{e}$ Series, 102 (1978), 305-330.

[F] J. Fell, The dual spaces of $C^{*}$-algebras, Trans. Amer. Math. Soc., 94(1960), 365-403.

[Fo] J. Fox, Frobenius reciprocity and extensions of nilpotent Lie groups, Trans. Amer. Math. Soc., 298, no. 1 (Nov. 1986), 123-145.

[G-G-P] Gelfand, Graev, Pyateskii-Shapiro, Representation Theory and Automorphic Functions, W. B. Saunder, Philadelphia, 1969. 
[H1] R. Howe, On Frobenius reciprocity for unipotent algebraic groups over $Q$, Amer. J. Math., 93 (1971), 163-172.

[H2] _ Harmonic analysis on solvable algebraic groups, Pacific J. Math., 73 (1977), 383-437.

[K1] A. A. Kirillov, Unitary representations of nilpotent Lie groups, Russian Math. Surveys, 19 (1962), 53-104.

[K2] _ Elements of the Theory of Representations, Springer-Verlag, New York, 1976.

[Ma] G. Mackey, The Theory of Unitary Group Representations, University of Chicago Press, Chicago, 1976.

[M] C. Moore, Decomposition of unitary representations defined by discrete subgroups of nilpotent groups, Ann. of Math., 82 (1965), 146-182.

[M-W] C. Moore and J. Wolf, Square-integrable representations of nilpotent Lie groups, Trans. Amer. Math. Soc., 185 (1973), 445-462.

[M-T] G. Mostow and T. Tamagawa, The compactness of arithmetically defined homogeneous spaces, Ann. of Math., 76 (1962), 446-463.

[Pu1] L. Pukanszky, Leçons sur les répresentations des groupes, Dunod, Paris, 1967.

[Pu2] _ On the characters and Plancherel formula of nilpotent Lie groups, $\mathbf{J}$. Funct. Anal., 1 (1967), 225-280.

[Ra] M. Raghunatham, Discrete Subgroups of Lie Groups, Springer-Verlag, New York, 1972.

[R] L. Richardson, Decomposition of the $L^{2}$-space of a general compact nilmanifold, Amer. J. Math., 93 (1971), 173-189.

[S] M. Silman, Théorie de Mackey pour les groups adéliques, Asterisque, 115 (1984).

[T] T. Tamagawa, Adeles, in Algebraic Groups and Discontinuous Subgroups, edited by Borel, Mostow, Proceedings of Symposia in Mathematics, 9, Providence, American Math. Soc. (1966).

[Wa] N. Wallach, Symplectic Geometry and Fourier Analysis, Math. Science Press, Brookline, 1977.

[W-1] A. Weil, Basic Number Theory, Springer-Verlag, New York, 1974.

[W-2] _ Adeles and Algebraic Groups, Progress in Math., 23, Birkhauser, Boston, 1982.

Received January 11, 1988. Research partly supported by a LSU Summer Research Grant and by NSF Grant DMS-8703572. 


\section{PACIFIC JOURNAL OF MATHEMATICS EDITORS}

\author{
V. S. VARAdarajan \\ (Managing Editor) \\ University of California \\ Los Angeles, CA 90024-1555-05 \\ Herbert Clemens \\ University of Utah \\ Salt Lake City, UT 84112 \\ ThOMAS ENRIGHT \\ University of California, San Diego \\ La Jolla, CA 92093
}

R. FINN

Stanford University

Stanford, CA 94305

HeRmann FlaschKa

University of Arizona

Tucson, AZ 85721

VAUGHAN F. R. Jones

University of California

Berkeley, CA 94720

SteVen KercKhofF

Stanford University

Stanford, CA 94305

\section{ROBION KIRBY}

University of California

Berkeley, CA 94720

C. C. MOORE

University of California

Berkeley, CA 94720

HAROLD STARK

University of California, San Diego

La Jolla, CA 92093

\section{ASSOCIATE EDITORS}
R. Arens
E. F. BeCKenbaCH
B. H. NEUMANN
F. Wolf
K. YoshidA (1906-1982)

\section{SUPPORTING INSTITUTIONS}

UNIVERSITY OF ARIZONA

UNIVERSITY OF BRITISH COLUMBIA

CALIFORNIA INSTITUTE OF TECHNOLOGY

UNIVERSITY OF CALIFORNIA

MONTANA STATE UNIVERSITY

UNIVERSITY OF NEVADA, RENO

NEW MEXICO STATE UNIVERSITY OREGON STATE UNIVERSITY

\author{
UNIVERSITY OF OREGON \\ UNIVERSITY OF SOUTHERN CALIFORNIA \\ STANFORD UNIVERSITY \\ UNIVERSITY OF HAWAII \\ UNIVERSITY OF TOKYO \\ UNIVERSITY OF UTAH \\ WASHINGTON STATE UNIVERSITY \\ UNIVERSITY OF WASHINGTON
}

The Supporting Institutions listed above contribute to the cost of publication of this Journal, but they are not owners or publishers and have no responsibility for its content or policies.

Mathematical papers intended for publication in the Pacific Journal of Mathematics should be in typed form or offset-reproduced (not dittoed), double spaced with large margins. Please do not use built up fractions in the text of the manuscript. However, you may use them in the displayed equations. Underline Greek letters in red, German in green, and script in blue. The first paragraph must be capable of being used separately as a synopsis of the entire paper. In particular it should contain no bibliographic references. Please propose a heading for the odd numbered pages of less than 35 characters. Manuscripts, in triplicate, may be sent to any one of the editors. Please classify according to the scheme of Math. Reviews, Index to Vol. 39. Supply name and address of author to whom proofs should be sent. All other communications should be addressed to the managing editor, or Elaine Barth, University of California, Los Angeles, California 90024-1555-05.

There are page-charges associated with articles appearing in the Pacific Journal of Mathematics. These charges are expected to be paid by the author's University, Government Agency or Company. If the author or authors do not have access to such Institutional support these charges are waived. Single authors will receive 50 free reprints; joint authors will receive a total of 100 free reprints. Additional copies may be obtained at cost in multiples of 50 .

The Pacific Journal of Mathematics is issued monthly as of January 1966. Regular subscription rate: $\$ 190.00$ a year (5 Vols., 10 issues). Special rate: $\$ 95.00$ a year to individual members of supporting institutions.

Subscriptions, orders for numbers issued in the last three calendar years, and changes of address should be sent to Pacific Journal of Mathematics, P.O. Box 969, Carmel Valley, CA 93924, U.S.A. Old back numbers obtainable from Kraus Periodicals Co., Route 100, Millwood, NY 10546.

The Pacific Journal of Mathematics at P.O. Box 969, Carmel Valley, CA 93924 (ISSN 0030-8730) publishes 5 volumes per year. Application to mail at Second-class postage rates is pending at Carmel Valley, California, and additional mailing offices. Postmaster: send address changes to Pacific Journal of Mathematics, P.O. Box 969, Carmel Valley, CA 93924.

PUBLISHED BY PACIFIC JOURNAL OF MATHEMATICS, A NON-PROFIT CORPORATION Copyright (C) 1989 by Pacific Journal of Mathematics 


\section{Pacific Journal of Mathematics \\ Vol. 140, No. $2 \quad$ October, 1989}

Edoardo Ballico, Spanned and ample vector bundles with low Chern numbers

Marcy Mason Barge, Richard Swanson and Russell Bruce Walker,

Conjugacy class structure of smooth hyperbolic sectors . ........... 217

Jeffrey Stephen Fox, Adeles and the spectrum of compact nilmanifolds . . ..233

Robert D. Little, Homotopy complex projective spaces with divisible

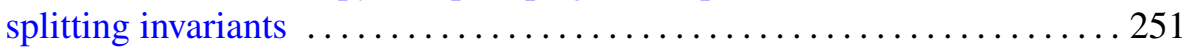

M. Scott Osborne and Garth William Warner, Jr., The Selberg trace formula. VII. Application of the truncation process to the continuous

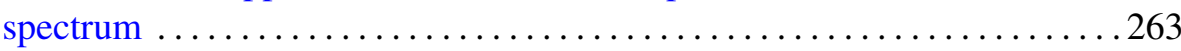

John R. Stembridge, On the eigenvalues of representations of reflection

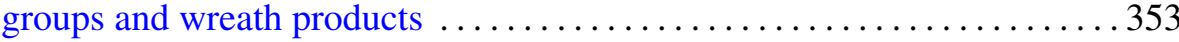

Ibrahim Salama, Corrections to: "Topological entropy and recurrence of

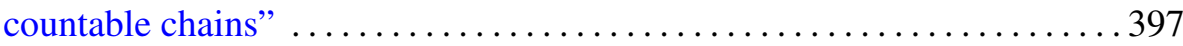

Robert Greene and Hung-Hsi Wu, Addendum to: "Lipschitz convergence of Riemannian manifolds" ............................... 398

Kayoko Shikishima-Tsuji, Correction to: "Galois theory of differential fields of positive characteristic" 